\title{
Sequence Analysis of Peptide:Oligonucleotide Heteroconjugates by Electron Capture Dissociation and Electron Transfer Dissociation
}

\author{
Kady L. Krivos and Patrick A. Limbach \\ Rieveschl Laboratories for Mass Spectrometry, Department of Chemistry, University of Cincinnati, \\ Cincinnati, Ohio, USA
}

\begin{abstract}
Mass spectrometry analysis of protein-nucleic acid cross-links is challenging due to the dramatically different chemical properties of the two components. Identifying specific sites of attachment between proteins and nucleic acids requires methods that enable sequencing of both the peptide and oligonucleotide component of the heteroconjugate cross-link. While collision-induced dissociation (CID) has previously been used for sequencing such heteroconjugates, CID generates fragmentation along the phosphodiester backbone of the oligonucleotide preferentially. The result is a reduction in peptide fragmentation within the heteroconjugate. In this work, we have examined the effectiveness of electron capture dissociation (ECD) and electron-transfer dissociation (ETD) for sequencing heteroconjugates. Both methods were found to yield preferential fragmentation of the peptide component of a peptide:oligonucleotide heteroconjugate, with minimal differences in sequence coverage between these two electron-induced dissociation methods. Sequence coverage was found to increase with increasing charge state of the heteroconjugate, but decreases with increasing size of the oligonucleotide component. To overcome potential intermolecular interactions between the two components of the heteroconjugate, supplemental activation with ETD was explored. The addition of a supplemental activation step was found to increase peptide sequence coverage over ETD alone, suggesting that electrostatic interactions between the peptide and oligonucleotide components are one limiting factor in sequence coverage by these two approaches. These results show that ECD/ETD methods can be used for the tandem mass spectrometry sequencing of peptide:oligonucleotide heteroconjugates, and these methods are complementary to existing CID methods already used for sequencing of protein-nucleic acid cross-links. (J Am Soc Mass Spectrom 2010, 21, 1387-1397) @ 2010 Published by Elsevier Inc. on behalf of American Society for Mass Spectrometry
\end{abstract}

$\mathrm{P}$ rotein-nucleic acid complexes have many diverse functions in cellular events such as pre-mRNA processing [1], RNA transcription [2], protein transportation [3], tRNA maturation [4], miRNA-based translational control [5], viral replication [6], and protein translation [7]. A traditional biochemical approach for the detailed structural analysis of protein-nucleic acid complexes has involved the use of cross-linking. Cross-linking studies provide information about specific interactions among the components of such complexes. An overriding characteristic of cross-linking studies has been the difficulties in elucidating specific protein and nucleic acid sites of interaction using conventional analytical or biochemical approaches [8].

Mass spectrometry has become a useful analytical approach for characterizing protein-nucleic acid crosslinked complexes [8,9]. Urlaub and coworkers have demonstrated the utility of mass spectrometry methods

Address reprint requests to Dr. P. A. Limbach, Rieveschl Laboratories for Mass Spectrometry, Department of Chemistry, University of Cincinnati, 429K Rieveschl P. O. Box 210172, University of Cincinnati, Cincinnati, OH 45221-0172, USA. E-mail: Pat.Limbach@uc.edu for cross-link analysis, exemplified by the characterization of small nuclear ribonucleoprotein (snRNP) complexes reconstituted in vitro and U1 snRNP complexes purified from HeLa cells [9-13]. Early work by this group utilized Edman degradation combined with matrix-assisted laser desorption/ionization mass spectrometry (MALDI-MS) to identify cross-linking sites on the protein component of the RNP complex [14, 15]. Another method for identification of cross-links took advantage of the oligonucleotide $3^{\prime}$ terminal phosphate that results after nuclease digestion of the cross-link [11]. Samples were digested by trypsin and divided in two, with one portion analyzed directly by MALDI-MS, and the second portion subjected to an additional alkaline phosphatase digestion step to remove any terminal phosphates before analysis by MALDI-MS. Cross-linked species were then identified by a mass shift of $79 \mathrm{Da}$ between the two samples.

More recently, liquid chromatography-mass spectrometry (LC-MS) methods have been developed for the characterization of protein-nucleic acid cross-links. One such development was the use of multiple reaction 
monitoring (MRM) scans designed to identify the loss of a phosphoric acid group $\left(\mathrm{HPO}_{3},-80 \mathrm{Da}\right)$, which serves as a signature for peptide:oligonucleotide crosslinks present in a mixture of peptides [13]. Ions detected by this MRM approach could be mapped onto the original complex by manually going through all possible combinations of tryptic peptides and digested nucleotides that could result in the MRM identified mass. Yet another method utilized the unique mass defect of peptide:oligonucleotide cross-links to differentiate between peptides and cross-links [16]. Because peptides contain a relatively high percentage of elements with a positive mass defect (e.g., $\mathrm{N}$ and $\mathrm{H}$ ) while oligonucleotides contain oxygen and phosphorus, which have negative mass defects, the use of high-resolution mass spectrometry, during LC-MS, allows one to identify mass defect values that are indicative of peptide:oligonucleotide cross-links.

In addition to methods that can be used to identify the presence of peptide:oligonucleotide cross-links within complex samples, Urlaub and coworkers have also developed purification methods compatible with downstream sequencing of such heteroconjugates [10]. Cross-links were first purified by an online 2-D nanoLC system integrated with $\mathrm{TiO}_{2}$ affinity purification. Purified cross-links were then identified using MALDI-MS and sequenced by either collision-induced dissociation (CID) on a tandem time-of-flight (TOF/TOF) [10] instrument or by post-source decay [12].

While those studies nicely illustrate the application of mass spectrometry for such structural studies, the sequence characterization of protein-nucleic acid complexes, usually as peptide:oligonucleotide cross-linked heteroconjugates, present a unique challenge to mass spectrometry because peptides and oligonucleotides prefer opposite ionization modes [17]. Jensen et al. first described the challenges of analyzing such heteroconjugates, and provided suggested instrumental conditions, spray solutions, and matrices that would be effective for either electrospray ionization (ESI) or MALDI mass spectrometry of heteroconjugates.

In addition to ionization challenges, mass spectrometrybased sequencing of heteroconjugates also presents difficulties arising from the different chemical properties of the oligonucleotide and peptide components. Most heteroconjugate sequencing by mass spectrometry is performed using CID as the method of fragmentation, where bond lability influences the fragmentation pathway [18, 19]. CID of peptide:oligonucleotide heteroconjugates produces more bond cleavages from the phosphodiester backbone of the oligonucleotide than from the peptide backbone [17]. Consequently, while CID (in particular, as implemented in LCMS/MS) is useful for identifying the sequence of the crosslinked oligonucleotide, along with the site of attachment in favorable cases, it is far less effective at reporting similar information for the peptide component. To overcome such limitations, most applications of CID of heteroconjugates require that the oligonucleotide component be minimized $[11,13,20,21]$.
Electron capture dissociation (ECD) and electrontransfer dissociation (ETD) are fragmentation methods in mass spectrometry that are complementary to CID methods for peptide and protein sequencing [22-25]. Unlike CID, in ECD and ETD bond strength does not dictate sites of fragmentation [26]. Although the exact mechanism is still subject to some debate, ECD and ETD fragmentation of peptides yields $c$ and $z$ series ions instead of $b$ and $y$ series ions as in CID [24, 25, 27].

Of particular interest here, these electron-based dissociation methods have been more effective at identifying sites of labile post-translational modifications, such as phosphorylations in proteins and peptides, than CIDbased approaches [28, 29]. Because peptide:oligonucleotide heteroconjugates can be viewed, simplistically, as peptides containing a labile modification (an oligonucleotide), we were interested in determining how effective ECD and/or ETD would be at generating fragmentation along the peptide backbone of a peptide:oligonucleotide heteroconjugate. Further, the effects of heteroconjugate charge state and size on ECD and ETD fragmentation were explored. We find that ECD and ETD can yield peptide fragmentation, useful for identifying sites of crosslink attachment on the peptide, and these sequencing approaches are complementary to CID-based sequencing of heteroconjugates. As with CID-based approaches, as the length of the oligonucleotide component increases, the reduction in cross-link charge state and/or intermolecular interactions between the peptide and oligonucleotide limit fragmentation efficiency. Supplemental activation during ETD was found to increase peptide fragmentation, suggesting that intermolecular interactions between the two components are one limiting factor in ECD and ETD efficiency.

\section{Materials and Methods}

\section{Materials}

The peptide, (Ac-GARGADRAVLARRR-NH $\mathrm{N}_{2}$, was purchased from Biomer Technology (Hayward, CA, USA), and was synthesized with an acetylated $\mathrm{N}$ terminus and an amidated C-terminus to avoid crosslinking at undesired points. A dinucleotide 5' $5^{\prime}$ - PUU-3' was obtained from Dharmacon RNAi Technologies (Lafayette, CO, USA) with a 6-carbon amino-linker on the $5^{\prime}$ phosphate group. Peptide-oligonucleotide heteroconjugate 2 (HC2), with the dinucleotide coupled to the carboxylic acid of the aspartic acid residue on the peptide via the amino-linker, was provided by Biomer Technology using the peptide and dinucleotide as starting materials. Peptide-oligonucleotide heteroconjugate 1 (HC1) was generated from $\mathbf{H C} 2$ by digestion with one unit of snake venom phosphodiesterase (SVP, Worthington Biochemical Corp., Lakewood, NJ, USA) at $\mathrm{pH} 10$ for $48 \mathrm{~h}$. Complete digestion of the terminal uridine was confirmed by MALDI-MS. 
Solvents for HPLC and ESI-MS were obtained from Tedia Company Inc. (Fairfield, OH, USA) or Honeywell Burdick and Jackson (Muskegon, MI, USA). Triethylamine and formic acid were purchased from Fisher Scientific (Pittsburg, PA, USA), and ammonium acetate was purchased from EMD (Gibbstown, NY, USA).

\section{Heteroconjugate Purification}

HC2 was purified by HPLC to remove unreacted peptide and oligonucleotide. A Hitachi LaChrome Elite HPLC (Hitachi, San Jose, CA, USA) with a L2130 pump/controller and L-2455 diode array detector was used. The column used was a $5 \mu \mathrm{m} 4.6 \mathrm{~mm} \times 250 \mathrm{~mm}$ Supelcosil LC-18 column (Sigma-Aldrich, St. Louis, MO, USA). Buffer A was $5 \mathrm{mM}$ ammonium acetate with $0.1 \%$ formic acid, and Buffer B was $100 \%$ acetonitrile with $0.1 \%$ formic acid. A linear gradient was used from $1 \%$ B to $90 \%$ B over 50 min. One-minute fractions were collected, taken to dryness and resuspended with 50\% aqueous acetonitrile, $5 \mathrm{mM}$ ammonium acetate, and $0.1 \%$ formic acid. The fraction containing the cross-link was identified by MALDI-MS, and then stored at $-80{ }^{\circ} \mathrm{C}$ before analysis.

To purify $\mathbf{H C 1}$ from the enzymatic digestion of $\mathbf{H C 2}$, the digestion mixture was loaded on a 50,000 Da molecular weight cut-off filter from Amicon (Millipore, Billerica, MA, USA) to remove SVP. The sample was then desalted with Millipore C18 Zip-Tips and stored at $0{ }^{\circ} \mathrm{C}$ before analysis.

\section{Mass Spectrometry}

All MALDI-TOFMS analyses were performed on a Bruker (Billerica, MA, USA) Reflex IV equipped with a nitrogen laser and operated in positive polarity, reflectron mode. Matrix was prepared using a saturated solution of 2,4,6-trihydroxyacetophenone in $50 \mathrm{mM}$ diammonium hydrogen citrate $/ 50 \%$ aqueous acetonitrile. Approximately $1 \mu \mathrm{L}$ of sample was combined with $5 \mu \mathrm{L}$ of matrix and spotted on a MTP 384 Massive target plate and allowed to dry.

All ECD and CID experiments were performed in positive polarity on a Thermo (Waltham, MA, USA) LTQ-FTMS. Samples were diluted into a buffer of 50\% aqueous acetonitrile, $5 \mathrm{mM}$ ammonium acetate and $0.1 \%$ formic acid then loaded into PicoTip (New Objective, Woodburn, MA, USA) $2 \pm 1 \mu \mathrm{m}$ emitters for static nanospray. The general parameters used at the spray interface were a capillary voltage of $35 \mathrm{~V}$, capillary temperature of $275^{\circ} \mathrm{C}$ and a tube lens of $110 \mathrm{~V}$. ECD energies were varied from 0 to 20 in arbitrary units (a.u.) with durations ranging from 7 to $120 \mathrm{~ms}$. A normalized collision energy of $20 \%-50 \%$ was used for CID experiments. The tip voltage was typically $1.5 \mathrm{kV}$ and isolation widths were typically $2--5 \mathrm{~m} / \mathrm{z}$ units. All samples, peptides and heteroconjugates, were subjected to the same CID and ECD conditions to facilitate comparisons of fragmentation.
All ETD experiments were performed in positive polarity on a Thermo LTQ-XL using fluoranthene as the anion reagent. Samples were diluted into a buffer of $50 \%$ aqueous acetonitrile, $5 \mathrm{mM}$ ammonium acetate and $0.1 \%$ formic acid then loaded into PicoTip $2 \pm 1 \mu \mathrm{m}$ emitters for static nanospray. The general parameters used at the spray interface were a capillary voltage of 30-40 V, capillary temperature of $200{ }^{\circ} \mathrm{C}$, and a tube lens of 100-200 V. ETD durations were varied from 0-200 ms, which were obtained by automatic optimization on a known ETD fragment. The tip voltage was typically $1.5 \mathrm{kV}$ and isolation widths were typically $2-5$ $\mathrm{m} / \mathrm{z}$ units. Default supplemental activation (SA) conditions were used for all ETD-SA experiments.

\section{Results and Discussion}

ECD and ETD are effective dissociation approaches for localizing sites of phosphorylation in peptide sequences $[29,30]$. Because peptide phosphorylation can be viewed as a simplistic example of a peptide:oligonucleotide heteroconjugate, the effectiveness of ECD and ETD for heteroconjugate sequence analysis was examined. Two heteroconjugates (Table 1) were used to assess the effects of charge state and length of the oligonucleotide on ECD and ETD efficiency. Results obtained using ECD and ETD were also compared with dissociation of these heteroconjugates using CID.

Before evaluating the effectiveness of ECD and ETD at sequencing heteroconjugates, the 14 amino acid peptide (Ac-GARGADRAVLARRR- $\mathrm{NH}_{2}$ ), without a conjugated mono- or dinucleotide, was characterized by CID, ECD and ETD (Supplemental Figure S1, which can be found in the electronic version of this article). This peptide was used as a model system because it allowed for a direct comparison to previous results obtained by Jensen et al. on this peptide and subsequent peptide: oligonucleotide heteroconjugates [17]. Fragmentation of the $3+$ charge state (the most abundant charge state) resulted in 12 out of 26 expected $b$ and $y$ series ions for CID (Supplemental Figure S1a), 23 out of 26 expected $c$ and $z$ series ions for ECD (Supplemental Figure S1b), and 17 out of 26 expected $c$ and $z$ series ions for ETD (Supplemental Figure S1c). These fragmentation data serve as the reference point to compare whether dissociation of a heteroconjugate is comparable to dissociation of the peptide alone.

Table 1. Peptide-oligonucleotide heteroconjugates (HC) investigated in this study

\begin{tabular}{lc}
\hline \multicolumn{1}{c}{ Abbreviation } & \multicolumn{1}{c}{ Sequence } \\
\hline \hline Heteroconjugate & Ac-GARGAD $\left(\mathrm{NH}\left(\mathrm{CH}_{2}\right)_{6} \mathrm{pC}\right)$ RAVLARRR-NH \\
1 (HC1) & \\
Heteroconjugate & $\mathrm{Ac}-\mathrm{GARGAD}\left(\mathrm{NH}\left(\mathrm{CH}_{2}\right)_{6} \mathrm{pCpU}\right)$ RAVLARRR- \\
2 (HC2) & $\mathrm{NH}_{2}$ \\
\hline
\end{tabular}




\section{CID, ECD, and ETD of HC1}

HC1 is a heteroconjugate comprised of a 14 amino acid peptide containing 5 arginine residues covalently linked through an internal aspartic acid residue to a single cytidine $5^{\prime}$-monophosphate. The ESI mass spectrum of HC1 (Supplemental Figure S2a) shows a charge distribution that favors the $3+$ charge state with an appreciable fraction in the $2+$ and $4+$ charge states. CID of the $3+$ charge state only yields one $d$ series nucleotide fragment with no major fragment ions that can be assigned to the peptide backbone (Supplemental Figure S2b). Figure $1 \mathrm{a}$ and $\mathrm{b}$ are representative data obtained from ECD and ETD of the 3+ charge state, respectively. ECD generates 21 out of 26 expected $c$ and $z$ series peptide backbone fragments, one $d$ series nucleotide fragment at two charge states, and one arginine side-chain loss (Figure 1a). ETD produces 19 out of 26 expected $c$ and $z$ series peptide backbone fragments (Figure $1 b$ ). The sequence coverage from ECD is sufficient to localize the cytidine $5^{\prime}$-monophosphate site of attachment to the aspartic acid residue, while ETD results are sufficient to localize it to one of two amino acids in the peptide sequence. Thus, ECD and ETD fragmentation of $\mathbf{H C 1}$ provides essentially identical sequence coverage as ECD and ETD fragmentation of the peptide component lacking the aminohexyl-linked mononucleotide.

Similar results were obtained from CID, ECD and ETD fragmentation of the $4+$ charge state of HC1. CID of HC1 (Supplemental Figure S3a) yields fragmentation localized to the cytidine phosphodiester group with no fragmentation detected along the peptide backbone. In contrast, both ECD (Supplemental Figure S3b) and ETD (Supplemental Figure S3c) yield significant fragmentation along the peptide backbone. ECD provides 22 out of 26 expected $c$ and $z$ series ions from the peptide backbone and one $d$ series ion arising from the cytidine phosphodiester group. Similarly, ETD provides 21 out of 26 expected $c$ and $z$ series ions from the peptide backbone. This level of sequence coverage from both ECD and ETD is sufficient to localize the attachment site to the aspartic acid residue.

Although the 2+ charge state for $\mathbf{H C 1}$ was generated at a significantly lower abundance than the $3+$ charge state, CID, ECD and ETD characterization of this charge state was also attempted resulting in only minor sequence coverage of the peptide backbone. Again, CID results in fragmentation only at the cytidine phosphodiester group (Supplemental Figure S4a). ECD yielded 4 out of 26 expected $c$ and $z$ series ions from the peptide backbone (Supplemental Figure S4b). The ETD results from the 2+ charge state of $\mathbf{H C 1}$ are discussed in a later section.

Sequence characterization of the $4+$ charge state of HC1 is enhanced by the larger electron capture crosssection, which leads to a relatively high signal-to-noise ratio for fragment ions generated by ECD or ETD. The only significant drawback to working with a highly charged precursor is that the product ions have charge states ranging from $1+$ to $3+$, which can hinder sequence assignments in cases where the charge state cannot be unambiguously determined. As the 3+ charge state was generated at a similar, albeit slightly higher, abundance as the $4+$ charge state, the signal-tonoise ratios of product ions from dissociation of the $3+$ charge state were generally sufficient for routine assignment with product ion charge states detected at both $1+$ and $2+$.

\section{CID, ECD, and ETD of HC2}

The results obtained from HC1 suggest that ECD and ETD are potentially effective dissociation methods for generating peptide backbone cleavages that would permit the localization of a site of cross-linking within a peptide:oligonucleotide heteroconjugate. Moreover, ECD and ETD were both found to be significantly better than CID, which did not yield any appreciable peptide backbone fragment ions. However, HC1 contained only a simple mononucleotide covalently attached to the peptide through the aminohexyl-linker. To determine whether the addition of another nucleotide group would affect ECD and ETD fragmentation of the backbone, a second heteroconjugate, HC2, was analyzed. The only difference between HC1 and HC2 is an additional uridine $5^{\prime}$-monophosphate onto the $3^{\prime}$-end of the cytidine, creating a dinucleotide covalently attached to the peptide through the aminohexyl-linkage at the internal aspartic acid.

The ESI mass spectrum of HC2 is seen in Supplemental Figure S5a and shows a charge distribution that favors the $3+$ charge state with an appreciable fraction in the $4+$ charge state. CID of HC2 generates two $d$ series fragments from the dinucleotide and no assignable peptide backbone fragment ions (Supplemental Figure S5b). Figure $2 \mathrm{a}$ and $\mathrm{b}$ are representative data obtained from ECD and ETD of the 3+ charge state, respectively. ECD yielded 10 out of 26 expected $c$ and $z$ series ions from the peptide backbone, and one $d$ series fragment from the dinucleotide (Figure 2a). ETD was discovered to be slightly more effective than ECD, as 13 out of 26 expected $c$ and $z$ series ions from the peptide backbone were detected (Figure $2 b$ ). Neither ECD nor ETD of the $3+$ charge state generated sufficient peptide backbone sequence coverage to uniquely locate the site of dinucleotide linkage, although both approaches could minimize the potential sites of cross-linking and both approaches were significantly more effective at characterizing the peptide component than was CID.

More peptide backbone cleavages were obtained from ECD and ETD fragmentation of the $4+$ charge state, as compared to the more abundant $3+$ charge state, of HC2. CID of the $4+$ charge state of HC2 generates only fragmentation along the dinucleotide backbone (Supplemental Figure S6a). ECD yielded 11 out of 26 expected $c$ and $z$ series ions from the peptide backbone (Supplemental Figure S6b). ETD yielded the 
(a)
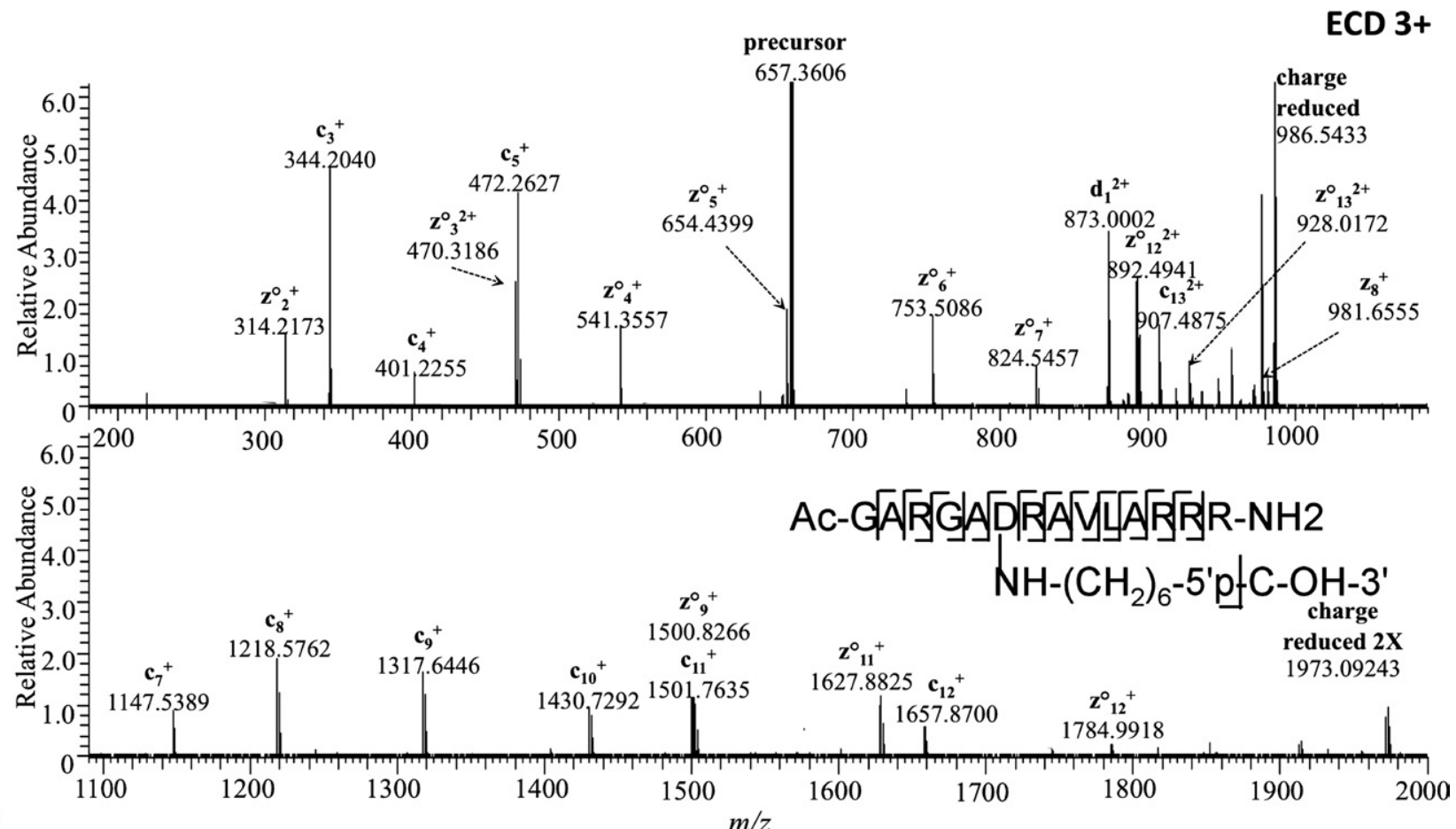

ETD 3+

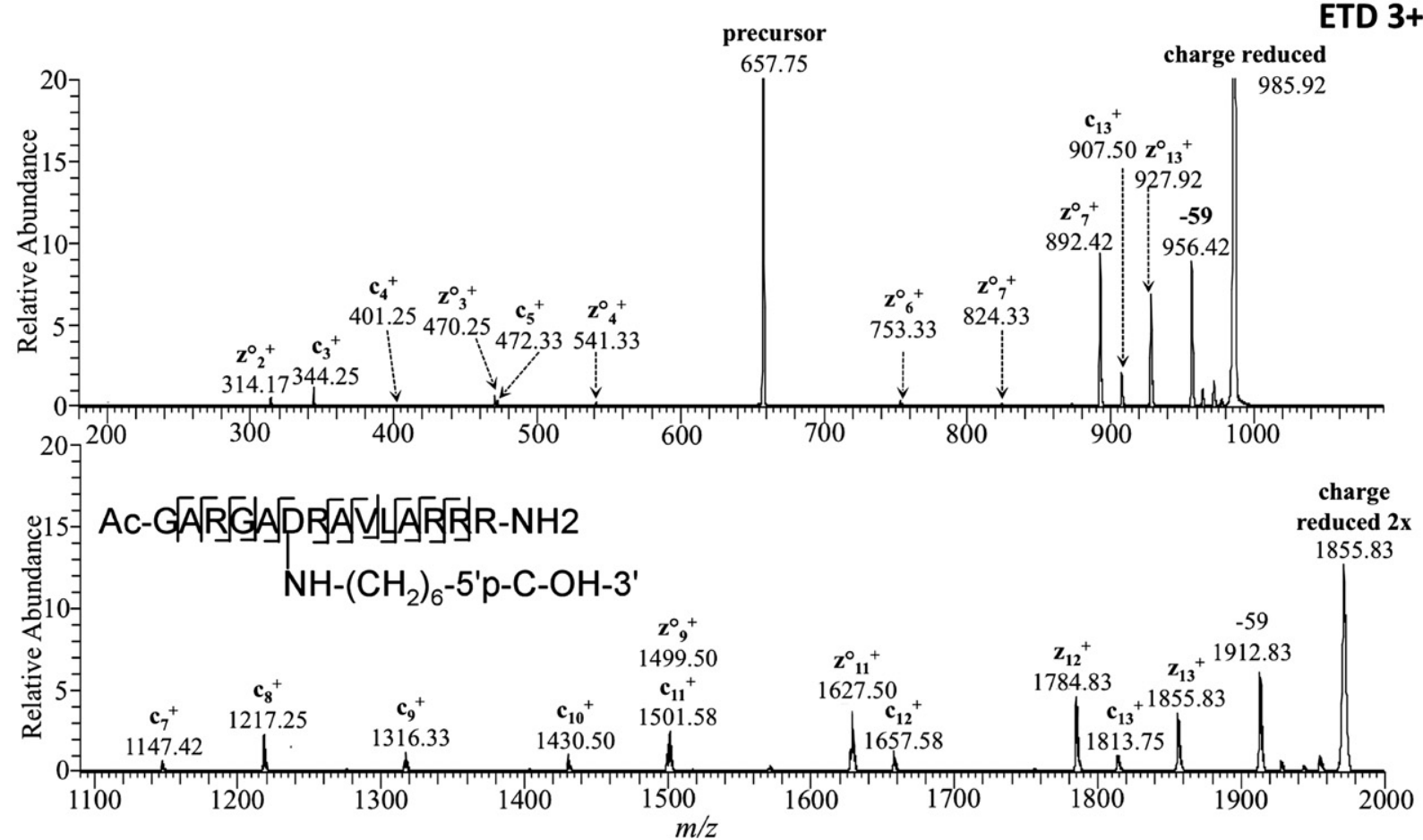

Figure 1. (a) ECD and (b) ETD tandem mass spectra of the $3+$ charge state for HC1. ECD parameters were: collision energy of 10 (a.u.) and duration of $70 \mathrm{~ms}$ with 120 summed scans. ETD parameters were: duration of $132 \mathrm{~ms}$ with 67 summed scans. Identified fragment ions are denoted on the mass spectra and mapped to the heteroconjugate sequence.

greatest number of backbone cleavages, with 22 out of 26 expected $c$ and $z$ series ions from the peptide backbone detected (Supplemental Figure S6c). The ETD results are sufficient to identify the amino acid with the aminohexyl linker.
CID, ECD, and ETD characterization of the low abundance $2+$ charge state was also attempted, although essentially no fragmentation of the peptide backbone resulted (Supplemental Figure S7). As with the $3+$ and $4+$ charge states, CID of the $2+$ charge state 

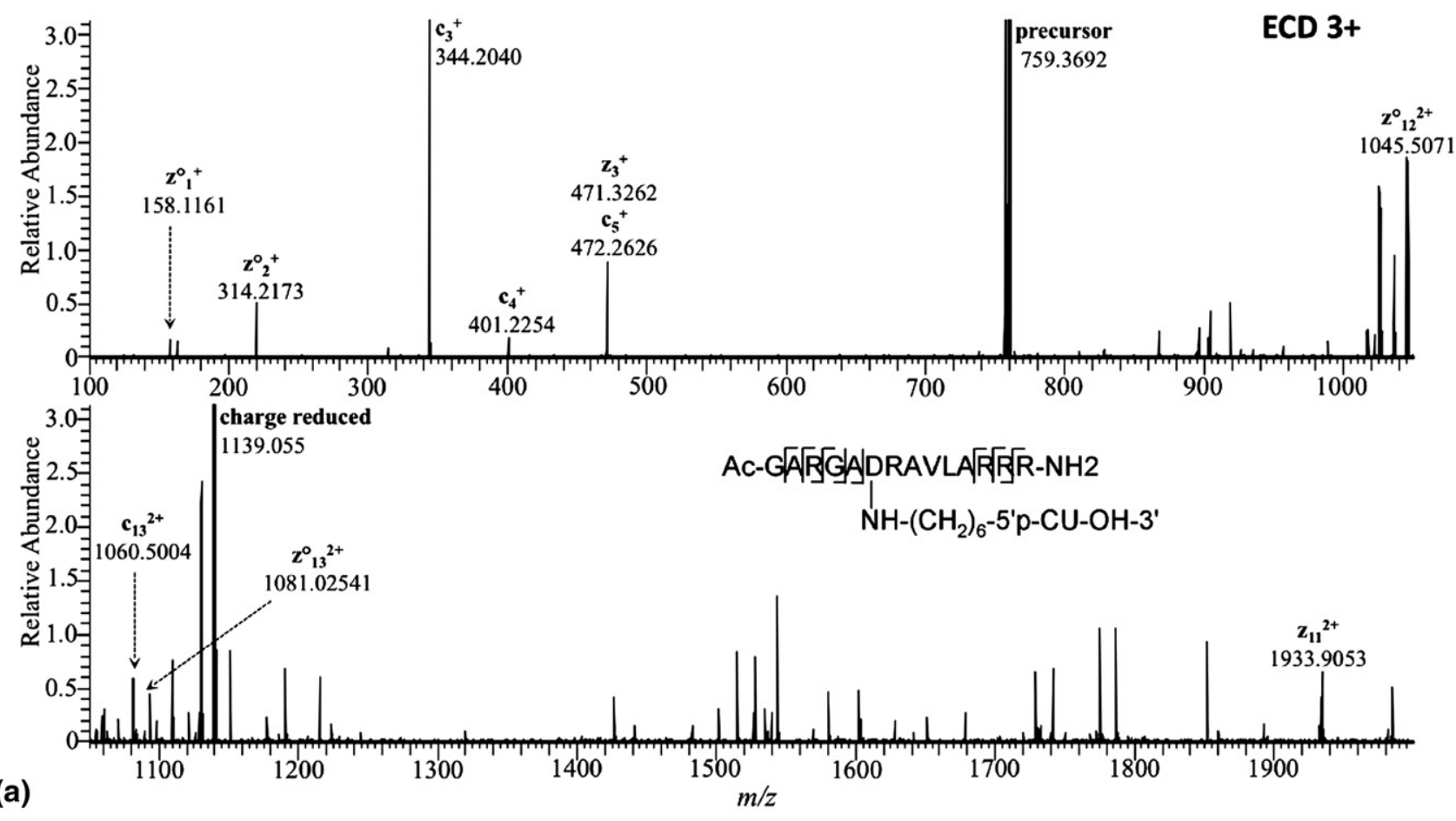

ETD 3+

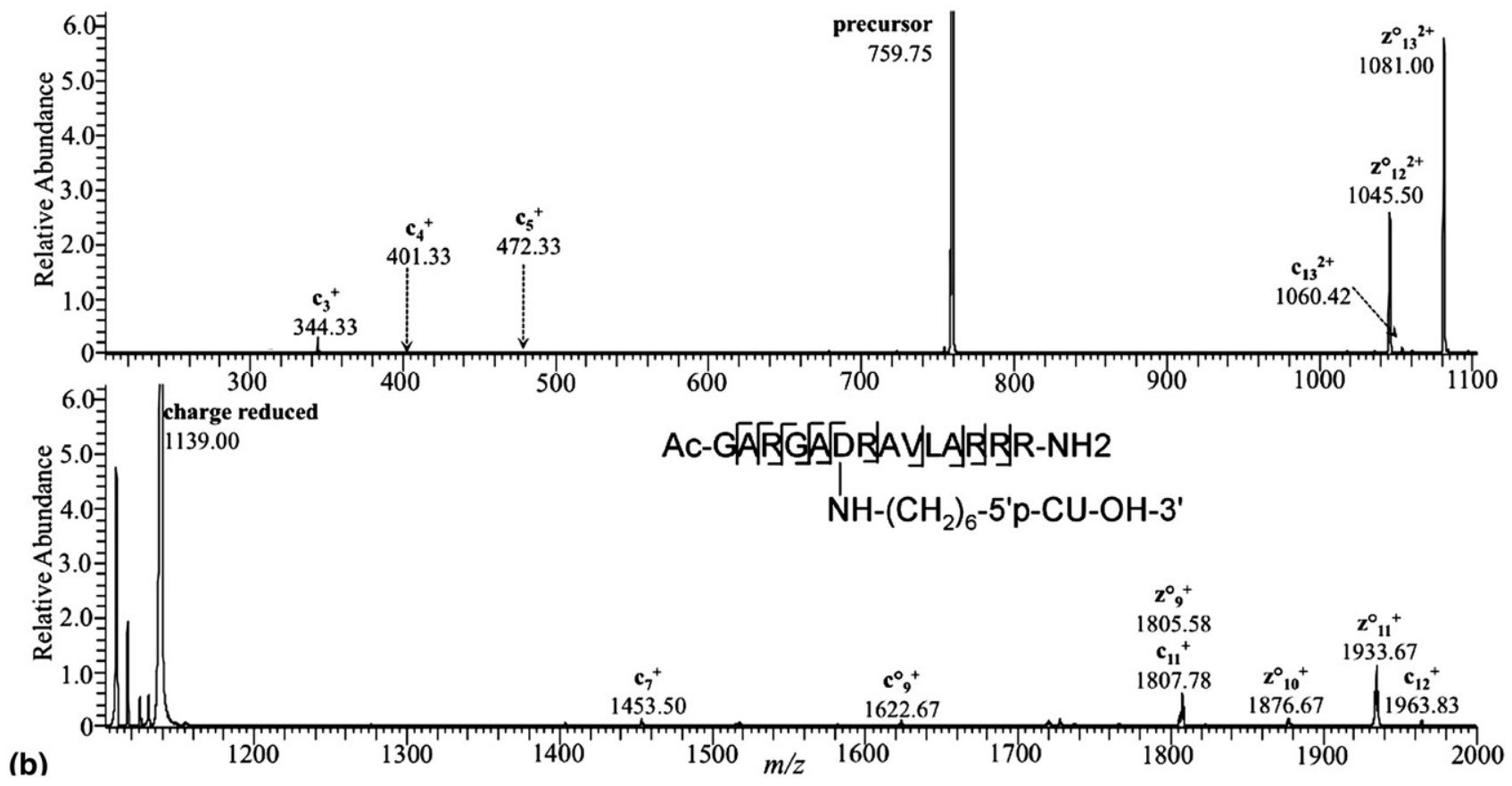

Figure 2. (a) ECD and (b) ETD tandem mass spectra of the $3+$ charge state for HC2. ECD parameters were: collision energy of 10 (a.u.) and duration of $70 \mathrm{~ms}$ with 170 summed scans. ETD parameters were: duration of $54 \mathrm{~ms}$ with 103 summed scans. Identified fragment ions are denoted on the mass spectra and mapped to the heteroconjugate sequence.

of $\mathrm{HC} 2$ results in fragmentation for only the dinucleotide (Supplemental Figure S7a). ECD of the $2+$ charge state yielded only the $\mathrm{z}_{11}{ }^{+}$ion that could be assigned to peptide fragmentation (Supplemental Figure S7b). In the case of ETD of the $2+$ charge state, no peptide or dinucleotide fragment ions were detected (Supplemental Figure S7c).

\section{Effect of Charge State on Heteroconjugate Fragmentation}

The charge state of peptide ion being investigated by ECD and ETD has been shown to play an important role in the efficiency of capturing electrons, with the probability of electron capture proportional to the square of 
the charge on the peptide [25]. Similar results were observed here with heteroconjugates HC1 and HC2. Table 2 summarizes the number of peptide fragment ions detected for both heteroconjugates at the $2+, 3+$, and $4+$ charge states along with the precursor ion abundance. For both heteroconjugates, minimal peptide fragment ions were detected at the $2+$ charge state. For HC1 the $3+$ and $4+$ charge states yielded comparable numbers of peptide fragment ions. Similarly, for HC2 the $3+$ and $4+$ charge states yielded comparable numbers of peptide fragment ions, except in the case of ETD of $\mathbf{H C} 2$ at the $4+$ charge state. ETD fragmentation at the $4+$ charge state for $\mathbf{H C} 2$ was more extensive than at the $3+$ charge state, with the number of peptide fragment ions detected being comparable to that found from ECD and ETD of HC1 at the $3+$ and $4+$ charge states.

No differences in peptide fragmentation that could be attributed to precursor ion abundance could be discerned from these experiments. Product ion abundances are similar for both heteroconjugates at all charge states between the two fragmentation techniques (ECD and ETD) indicating that the sensitivities of ECD and ETD for heteroconjugate sequencing are comparable. There are differences in product ion abundances when comparing the two heteroconjugates against each other. $\mathbf{H C} 1$ yields fragment ions that are 5 to 20-fold more abundant than fragment ions generated from HC2. Such differences may be due to the additional nucleotide residue present in $\mathbf{H C 2}$ as discussed further below.

To determine whether there are any differences in the sites of fragmentation of the peptide backbone during ECD and ETD of differing charge states, Figure 3 summarizes the results for Table 2 mapped on the heteroconjugate sequence. For $\mathrm{HC} 1$, the sites of fragmentation are consistent between the $3+$ and $4+$ charge states in both ECD and ETD (Figure $3 a$ and $b$ ). Peptide bond fragmentation occurs on both the $\mathrm{N}$ - and Cterminal regions of the peptide with fragmentation being reduced around the aspartic acid residue where the aminohexyl cross-linker is attached. For HC2 there is still a clear preference for peptide fragmentation near the $\mathrm{N}$ - and C-terminal regions, although this localization of fragmentation is more pronounced for ECD (Figure 3c) than ETD (Figure 3d). Both ECD and ETD result in fewer peptide fragments arising from the C-terminal side of the cross-link, signifying the possibility that there may be some secondary interactions between this positively charged region of the peptide (due to the three arginine residues) and the dinucleotide cross-link.

\section{ETD of Heteroconjugates with Supplemental Activation}

Although HC1 and HC2 showed an increase in the number of peptide fragment ions with an increase in charge state, at the $2+$ and $3+$ charge states, HC1 yielded more peptide fragments than HC2 when analyzed at the same charge state even though the only difference between these two heteroconjugates is an additional uridine nucleotide on HC2. One explanation for this difference could be that the dinucleotide serves as a more effective radical trap than the mononucleotide, thereby limiting these radical-driven fragmentation reactions [31]. Another of the possible explanations for this trend could be an increase in noncovalent interactions between the dinucleotide and peptide present in HC2 over the mononucleotide and same peptide present in HC1. Creese et al. previously showed that multiple phosphorylations lead to an increase in secondary interactions between the phosphate group and peptide for phosphopeptides, with these secondary interactions reducing ECD fragmentation [32].

Thus, additional experiments were attempted using supplemental activation with ETD (ETD-SA) in an attempt to increase peptide fragmentation for these two heteroconjugates. Figure 4 presents representative spectra from ETD and ETD-SA experiments on HC1 at the $2+$ charge state. ETD of this charge state results in only 2 out of 26 expected $c$ and $z$ series ions from the peptide backbone (Figure 4a). In contrast, ETD-SA of the $2+$ charge state generates 11 out of the 26 expected fragment ions with sequence coverage sufficient to localize the site of attachment to a three amino acid region of the peptide (Figure 4b). Thus, ETD-SA analysis of the heteroconjugates was expanded to all charge states of HC1 and HC2.

As seen in Table 2, for all charge states investigated $(2+, 3+$, and $4+)$, ETD-SA yielded a greater number of peptide fragments as compared to ETD alone for both HC1 and HC2. Interestingly, when the fragmentation results for the $2+$ and $3+$ charge states are mapped on the peptide sequence (Figure $3 \mathrm{e}-\mathrm{h}$ ), several trends in

Table 2. The number of peptide fragments out of the total possible fragments for each of the three cross-links at their available charge states for ECD and ETD along with the normalized intensities of the precursor ion

\begin{tabular}{|c|c|c|c|c|c|c|c|c|c|}
\hline & \multicolumn{3}{|c|}{$2+$} & \multicolumn{3}{|c|}{$3+$} & \multicolumn{3}{|c|}{$4+$} \\
\hline & ECD & ETD & ETD SA & ECD & ETD & ETD SA & ECD & ETD & ETD SA \\
\hline \multirow[t]{2}{*}{$\mathrm{HC} 1$} & $4 / 26$ & $2 / 26$ & $11 / 26$ & $21 / 26$ & $19 / 26$ & $20 / 26$ & $22 / 26$ & $21 / 26$ & $23 / 26$ \\
\hline & $8.0 \mathrm{E}+04$ & $2.3 E+04$ & $2.0 \mathrm{E}+04$ & $4.5 \mathrm{E}+06$ & $8.0 \mathrm{E}+04$ & $8.0 E+05$ & $2.0 \mathrm{E}+06$ & $6.0 \mathrm{E}+05$ & $6.0 \mathrm{E}+05$ \\
\hline \multirow[t]{2}{*}{$\mathrm{HC} 2$} & $1 / 26$ & $0 / 26$ & $6 / 26$ & $10 / 26$ & $13 / 26$ & $17 / 26$ & $11 / 26$ & $22 / 26$ & $24 / 26$ \\
\hline & $1.7 \mathrm{E}+05$ & $1.0 \mathrm{E}+04$ & $1.0 \mathrm{E}+04$ & $6.5 E+05$ & $3.5 E+05$ & $3.5 \mathrm{E}+05$ & $1.0 \mathrm{E}+05$ & $1.5 \mathrm{E}+04$ & $1.5 \mathrm{E}+04$ \\
\hline
\end{tabular}




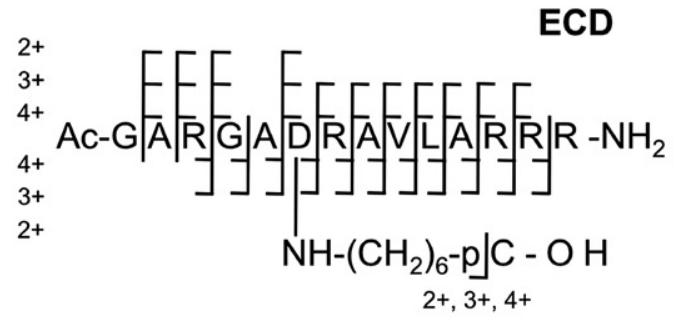

(b)

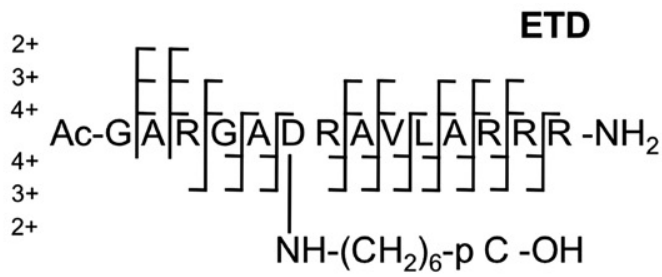

(a)

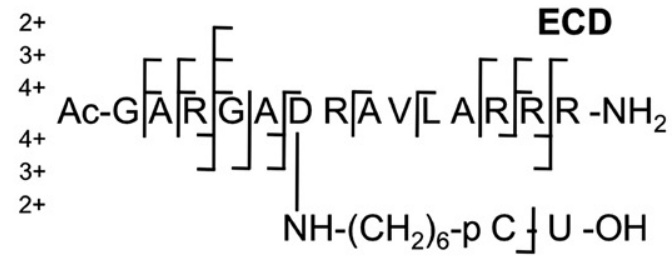

(c)

$$
2+, 3+, 4+
$$

2+

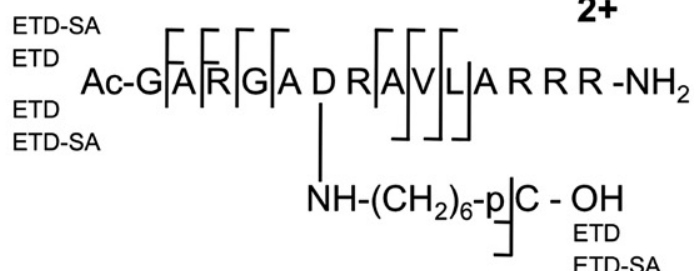

2+

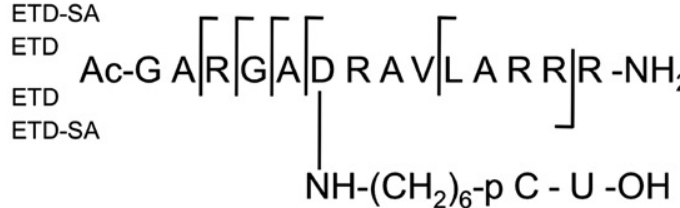

(g) (d)

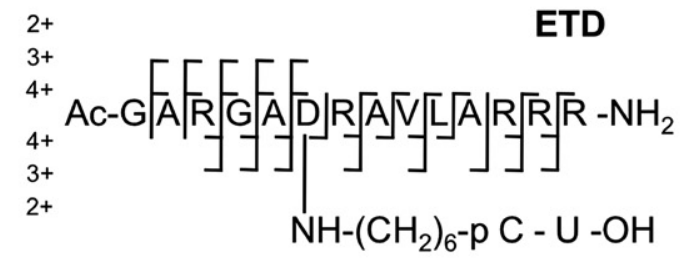

(f)

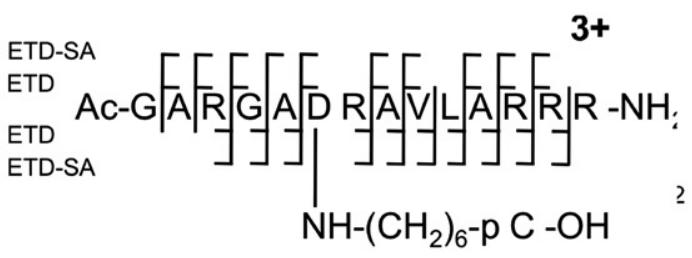

(h)
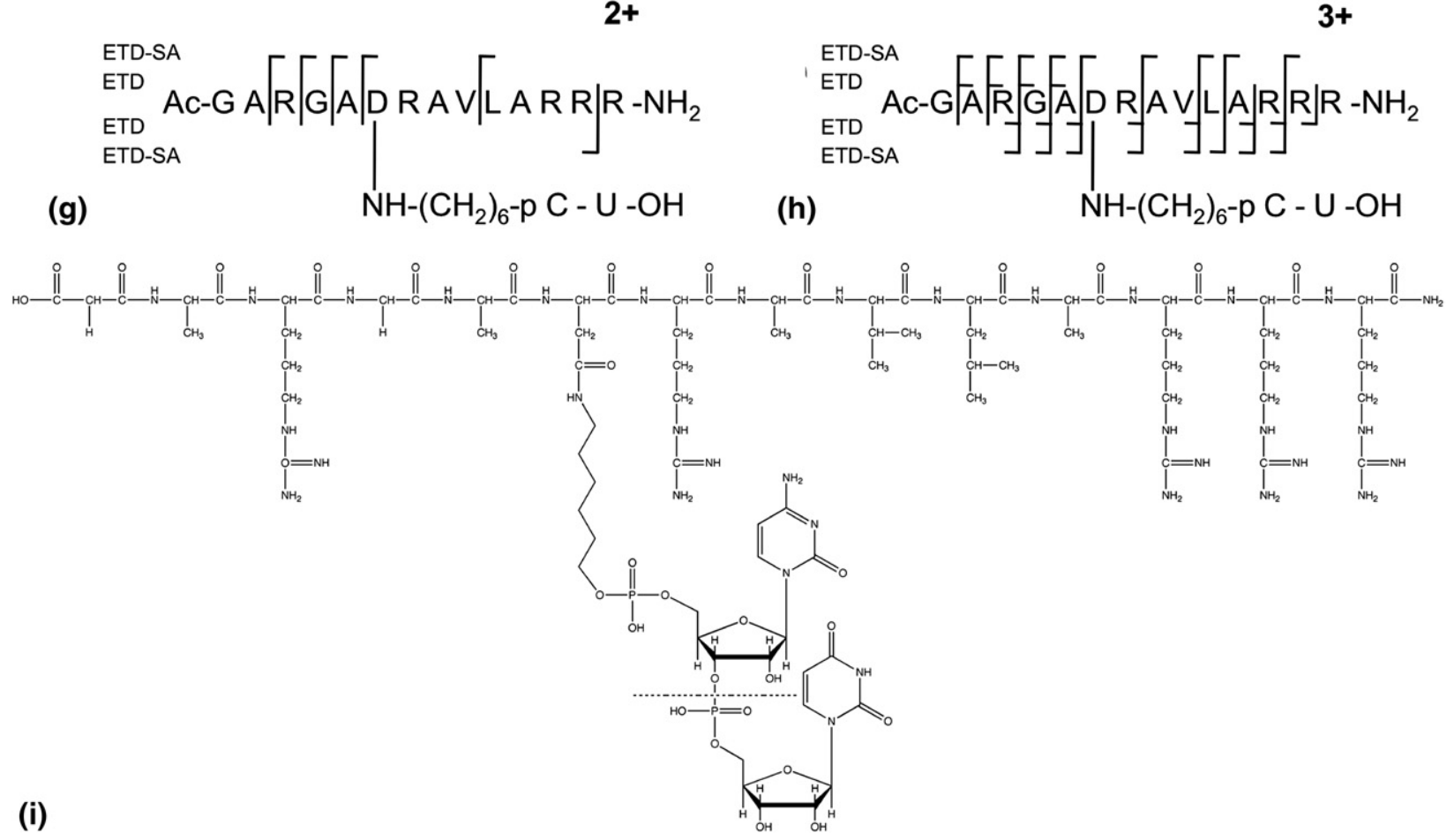

Figure 3. Sequence map of (a) ECD and (b) ETD $c$ - and $z$-series peptide fragments of HC1 at the 2+, $3+$, and $4+$ charge states. Sequence map of (c) ECD and (d) ETD $c$ - and $z$-series peptide fragments of HC2 at the 2+, 3+, and 4+ charge states. Sequence map comparison of ETD and ETD-SA $c$ - and $z$-series peptide fragments of $\mathbf{H C 1}$ at the (e) $2+$ and (f) $3+$ charge states. Sequence map comparison of ETD and ETD-SA $c$ - and $z$-series peptide fragments of HC2 at the (g) 2+ and (h) 3+ charge states. (i) Structure of HC2 (with HC1 arising from the loss of the uridine nucleotide). Comparative data of ETD and ETD-SA for the 4+ charge states of HC1 and HC2 are not shown due to minimal differences in the number of peptide fragment ions detected. For all figures, $c$-series fragment ions are shown to the bottom of the peptide sequence; $z$-series fragment ions are shown to the top of the peptide sequence; and, when detected, nucleotide fragment ions are shown. 
ETD 2+

(a)
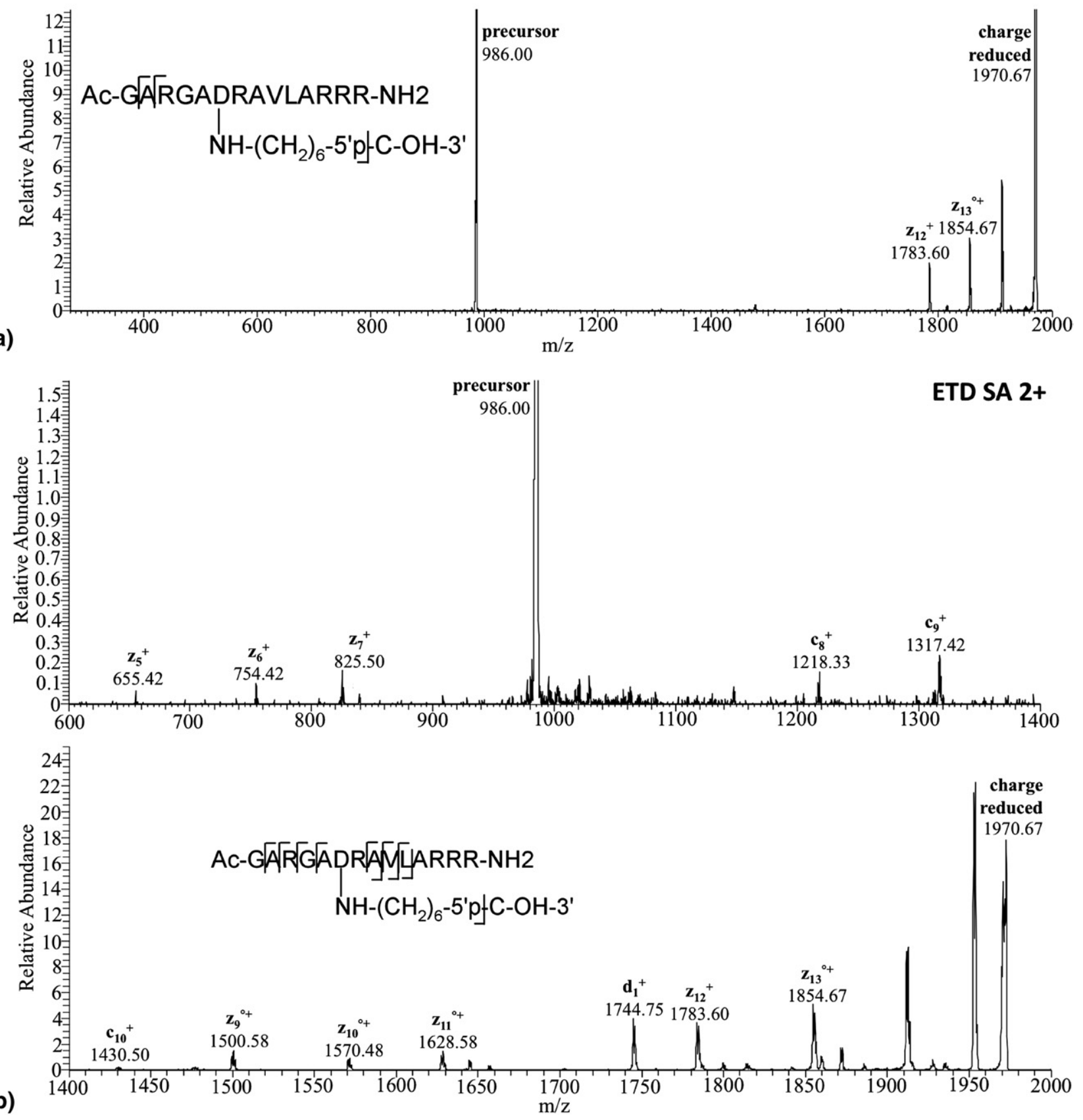

Figure 4. (a) ETD and (b) ETD-SA fragmentation of HC1 at the 2+ charge state. ETD and ETD-SA were performed for $200 \mathrm{~ms}$ and 20 scans were summed to generate the mass spectra shown. Fragment ions are identified and mapped to the heteroconjugate sequence. Similar increases in peptide fragmentation when adding supplemental activation were found at other charge states and when HC2 was analyzed.

the data comparing ETD versus ETD-SA fragmentation are noticed. The use of supplemental activation for both heteroconjugates generates significant peptide fragmentation on the N-terminal side of the site of attachment, which is also the N-terminal region of the peptide. These data are consistent with the results shown in Figure $3 a-d$, where more significant fragmentation occurs on the N-terminal region of the peptide for ECD and ETD at the various charge states investigated. In addition, an increase in peptide fragmentation on the C-terminal side of the site of attachment is found, with more significant increases noted for the $2+$ charge states of both heteroconjugates. Consistent with the discussion above, secondary interactions through salt bridges between the phosphodiester group and the positively-charged arginine residues may limit peptide 
fragmentation on the C-terminal region of the peptide (Figure 3i). Supplemental activation may, therefore, disrupt such interactions leading to the increase in peptide fragmentation in this region. Finally, the effect of supplemental activation is less pronounced for HC2 (the dinucleotide) than HC1 (the mononucleotide), which may also reflect additional sites of secondary interaction between the two components. Alternatively, electrostatic attraction between the positively charged C-terminus and the nucleotide component may allow the nucleotide component to serve as a radical trap inhibiting fragmentation along this region, with the dinucleotide serving as a stronger radical trap than the mononucleotide. While these data demonstrate supplemental activation increases peptide fragmentation for both heteroconjugates, additional experiments are required to differentiate between the possible explanations for such an increase.

\section{Comparison with Prior Heteroconjugate Sequencing Results}

Urlaub and coworkers have demonstrated the capabilities of several mass spectrometry methods for sequencing peptide:oligonucleotide heteroconjugates. Using post-source decay with MALDI-TOFMS, they characterized several cross-links containing large peptides (11 to 23 amino acids) cross-linked to dinucleotides [12]. The site of attachment on the peptide was identified for one cross-link, and for two others the site was localized to one of two amino acids. They also noted that for larger oligonucleotides components in a cross-link, such as a 5-mer oligonucleotides attached to an 11 amino acid residue peptide, only the oligonucleotide portion could be sequenced by post-source decay.

In other data obtained using a Q-TRAP platform, they found that MS/MS could be used to localize the region of attachment between oligonucleotide and peptide crosslinks, although the precise sites of attachment could not be determined [13]. Similarly, when using MALDI-TOF/ TOF as the mass spectrometry platform, they again could localize the region of cross-linking on the peptide component of the cross-link, with complete sequence coverage of the dinucleotide or trinucleotide component [10]. Therefore, the possibility exists that the CID conditions in the linear ion trap used in this study are less effective at generating fragmentation of the peptide component of these heteroconjugates. Whether instrumental differences account for the limitations of CID or there are unique factors arising from the cross-linker and/or heteroconjugate sequence will require further investigation.

\section{Conclusions}

The electron-induced dissociation methods ECD and ETD have been shown to be effective at generating fragmentation along the peptide component of a peptide-oligonucleotide heteroconjugate. In contrast to CID, which only generates fragmentation along the phosphodiester backbone of the oligonucleotide component, ECD and ETD generate peptide fragments that can be used to localize and/or identify sites of cross-linking to an oligonucleotide. Not surprisingly, peptide sequence coverage increases with increasing charge state of the heteroconjugate, with a $3+$ charge state being the minimal charge state for sufficient sequence coverage to localize a site of cross-linking.

Somewhat surprisingly, the addition of a single nucleotide to the oligonucleotide component of a heteroconjugate significantly reduced the number and abundance of peptide fragmentation when all other factors were equal. Thus, the possibility exists that the length of the oligonucleotide component may lead to increased non-covalent interactions between the peptide and oligonucleotide within the heteroconjugate. ETD-SA was shown to improve peptide fragmentation, particularly for lower charge state ions of a heteroconjugate having a longer oligonucleotide component. Although the present study was conducted using nanospray MS, ETD sequencing of heteroconjugates should be compatible with on-line LC-MS/MS analysis [33].

Based on these studies, several predictions regarding radical-driven fragmentation of peptide:oligonucleotide heteroconjugates can be made and tested. The acidic character of the oligonucleotide will inherently reduce the overall charge state of the heteroconjugate, which will prove challenging to these dissociation methods that benefit from more positively charged ions. Thus, compromises in the overall length of the oligonucleotide component may be necessary to ensure heteroconjugates are formed at the $3+$ charge state, as a minimum, as this charge state was found to yield significantly greater numbers of peptide fragment ions than the $2+$ charge state heteroconjugates. In addition, supplemental activation techniques for both ECD and ETD will be preferred to minimize secondary interactions (e.g., salt bridges) between the peptide and oligonucleotide components. Studies are currently underway to further clarify the role of the oligonucleotide and its secondary interactions with the peptide in the heteroconjugate during sequencing by ECD and ETD. Finally, for this particular model compound, the presence of a nonpolar methylene cross-linking reagent may also reduce fragmentation around the site of attachment to the peptide [34]. Thus, the type of cross-linking reagent may also affect the sequence coverage one can obtain from peptide:oligonucleotide heteroconjugates. Further studies comparing different cross-linking reagents, such as 4-thiouridine, 2-iminothiolane and platinum-based compounds are in progress.

\section{Acknowledgments}

The authors acknowledge financial support for this work provided by the National Institutes of Health (GM58843 and RR019900) and the University of Cincinnati. 


\section{Appendix A Supplementary Material}

Supplementary material associated with this article may be found in the online version at doi:10.1016/ j.jasms.2010.03.043.

\section{References}

1. Staley, J. P.; Woolford, J. L. J. Assembly of Ribosomes and Spliceosomes: Complex Ribonucleoprotein Machines. Curr. Opin. Cell. Biol. 2009, 21, 109-118.

2. Hager, G. L.; McNally, J. G.; Misteli, T. Transcription Dynamics. Molec. Cell. 2009, 35, 741-753.

3. Luirink, J.; Sinning, I. SRP-Mediated Protein Targeting: Structure and Function Revisited. Biochim. Biophys. Acta. 2004, 1694, 17-35.

4. Kazantsev, A. V.; Pace, N. R. Bacterial RNase P: A New View of an Ancient Enzyme. Nat. Rev. Microbiol. 2006, 4, 729-740.

5. Jackson, R. J.; Hellen, C. U. T.; Pestova, T. V. The Mechanism of Eukaryotic Translation Initiation and Principles of Its Regulation. Nat. Rev. Mol. Cell. Biol. 2010, 11, 113-127.

6. D'Souza, V.; Summers, M. F. How Retroviruses Select their Genomes. Nat. Rev. Microbiol. 2005, 3, 643-655.

7. Steitz, T. A. A Structural Understanding of the Dynamic Ribosome Machine. Nat. Rev. Mol. Cell. Biol. 2008, 9, 242-253.

8. Rusconi, F.; Guillonneau, F.; Praseuth, D. Contributions of Mass Spectrometry in the Study of Nucleic Acid-Binding Proteins and of Nucleic Acid-Protein Interactions. Mass Spectrom. Rev. 2002, 21, 305-348.

9. Urlaub, H.; Hartmuth, K.; Lührmann, R. A Two-Tracked Approach to Analyze RNA-Protein Crosslinking Sites in Native, Nonlabeled Small Nuclear Ribonucleoprotein Particles. Methods 2002, 26, 170-181.

10. Richter, F. M.; Hsiao, H.-H.; Plessmann, U.; Urlaub, H. Enrichment of Protein-RNA Crosslinks from Crude UV-Irradiated Mixtures for MS Analysis by On-Line Chromatography using Titanium Dioxide Columns. Biopolymers 2009, 91, 297-309.

11. Kühn-Holsken, E.; Dybkov, O.; Sander, B.; Lührmann, R.; Urlaub, H. Improved Identification of Enriched Peptide RNA Cross-links from Ribonucleoprotein Particles (RNPs) by Mass Spectrometry. Nucleic Acids Res. 2007, 35, e95.

12. Kühn-Hölsken, E.; Lenz, C.: Sander, B.; Lührmann, R. Urlaub, H. Complete MALDI-TOF MS Analysis of Cross-linked Peptide-RNA Oligonucleotides Derived from Nonlabeled UV-Irradiated Ribonucleoprotein Particles. RNA 2005, 11, 1915-1930.

13. Lenz, C.; Kühn-Hölsken, E.; Urlaub, H. Detection of Protein-RNA Cross-links by NanoLC-ESI-MS/MS Using Precursor Ion Scanning and Multiple Reaction Monitoring (MRM) Experiments. J. Am. Soc. Mass Spectrom. 2007, 18, 869-881.

14. Urlaub, H.; Hartmuth, K.; Kostka, S.; Grelle, G.; Lührmann, R. A General Approach for Identification of RNA-Protein Cross-Linking Sites Within Native Human Spliceosomal Small Nuclear Ribonucleoproteins (snRNPs). Analysis of RNA-Protein Contacts in Native U1 and U4/U6.U5 snRNPs. J. Biol. Chem. 2000, 275, 41458-41468.

15. Urlaub, H.; Raker, V. A.; Kostka, S.; Lührmann, R. Sm Protein-Sm Site RNA Interactions Within the Inner Ring of the Spliceosomal snRNP Core Structure. EMBO J. 2001, 20, 187-196.
16. Pourshahian, S.; Limbach, P. A. Application of Fractional Mass for the Identification of Peptide-Oligonucleotide Cross-links by Mass Spectrometry. J. Mass Spectrom. 2008, 43, 1081-1088.

17. Jensen, O. N.; Kulkarni, S.; Aldrich, J. V.; Barofsky, D. F. Characterization of Peptide-Oligonucleotide Heteroconjugates by Mass Spectrometry. Nucleic Acids Res. 1996, 24, 3866-3872.

18. Tromp, J. M.; Schürch, S. Gas-Phase Dissociation of Oligoribonucleotides and their Analogs Studied by Electrospray Ionization Tandem Mass Spectrometry. J. Am. Soc. Mass Spectrom. 2005, 16, 1262-1268.

19. Wells, M. J.; McLuckey, S. A. Collision-Induced Dissociation (CID) of Peptides and Proteins. In Methods in Enzymology: Biological Mass Spectrometry, Burlingame, A. L. Ed.; Academic Press: San Diego, CA, 2005; p. $148-185$.

20. Hanno, S.; Jørgen, P.; Matthias, M.; Jensen, O. N. Mass Spectrometric Analysis of a UV-Cross-Linked Protein-DNA Complex: Tryptophans 54 and 88 of E. coli SSB Cross-Link to DNA. Protein Sci. 2001, 10, 1989-2001.

21. Geyer, H.; Geyer, R.; Pingoud, V. A Novel Strategy for the Identification of Protein-DNA Contacts by Photocrosslinking and Mass Spectrometry. Nucleic Acids Res. 2004, 32, e132.

22. Cooper, H. J.; Hâkansson, K.; Marshall, A. G. The Role of Electron Capture Dissociation in Biomolecular Analysis. Mass Spectrom. Rev. 2005, 24, 201-222.

23. Zubarev, R. A. Reactions of Polypeptide Ions with Electrons in the Gas Phase. Mass. Spectrom. Rev. 2003, 22, 57-77.

24. Zubarev, R. A.; Horn, D. M.; Fridriksson, E. K.; Kelleher, N. L.; Kruger N. A.; Lewis, M. A.; Carpenter, B. K.; McLafferty, F. W. Electron Capture Dissociation for Structural Characterization of Multiply Charged Protein Cations. Anal. Chem. 2000, 72, 563-573.

25. Zubarev, R. A.; Kelleher, N. L.; McLafferty, F. W. Electron Capture Dissociation of Multiply Charged Protein Cations. A Nonergodic Process. I. Am. Chem. Soc. 1998, 120, 3265-3266.

26. Syrstad, E. A.; Tureček, F. Toward a General Mechanism of Electron Capture Dissociation. J. Am. Soc. Mass Spectrom. 2005, 16, 208-224.

27. Chamot-Rooke, J.; Malosse, C.; Frison, G.; Tureček, F. Electron Capture in Charge-Tagged Peptides. Evidence for the Role of Excited Electronic States. J. Am. Soc. Mass Spectrom. 2007, 18, 2146-2161.

28. Bakhtiar, R.; Guan, Z. Electron Capture Dissociation Mass Spectrometry in Characterization of Post-translational Modifications. Biochem. Biophys. Res. Commun. 2005, 334, 1-8.

29. Shi, S. D. H.; Hemling, M. E.; Carr, S. A.; Horn, D. M.; Lindh, I. McLafferty, F. W. Phosphopeptide/Phosphoprotein Mapping by Electron Capture Dissociation Mass Spectrometry. Anal. Chem. 2001, 73 19-22.

30. Mikesh, L. M.; Ueberheide, B.; Chi, A.; Coon, J. J.; Syka, J. E.; Shabanowitz, J.; Hunt, D. F. The Utility of ETD Mass Spectrometry in Proteomic Analysis. Biochim. Biophys. Acta 2006, 1764, 1811-1822.

31. Belyayev, M. A.; Cournoyer, J. J.; Lin, C.; O'Connor, P. B. The Effect of Radical Trap Moieties on Electron Capture Dissociation Spectra of Substance P. I Am. Soc. Mass Spectrom. 2006, 17, 1428-1436.

32. Creese, A. J.; Cooper, H. J. The Effect of Phosphorylation on the Electron Capture Dissociation of Peptide Ions. J. Am. Soc. Mass Spectrom. 2008, 19, 1263-1274.

33. Udeshi, N.; Shabanowitz, J.; Hunt, D.; Rose, K. Analysis of Proteins and Peptides on a Chromatographic Timescale by Electron-Transfer Dissociation MS. FEBS J. 2007, 274, 6269-6276.

34. Vorobyev, A.; Hamidane, H. B.; Tsybin, Y. O. Electron Capture Dissociation Product Ion Abundances at the X Amino Acid in RAAAA-XAAAAK Peptides Correlate with Amino Acid Polarity and Radical Stability. J. Am. Soc. Mass Spectrom. 2009, 20, 2273-2283. 